\title{
LONG TERM BEHAVIOUR OF PASSIVELY HEATED OR COOLED A-SI:H MODULES
}

\author{
C. Hof, M. Lüdi, M. Goetz, D. Fischer, A. Shah \\ Institute of Microtechnology, University of Neuchâtel, A.- L. Breguet 2, CH-2000 Neuchâtel, Switzerland \\ tel.: +41 -38 2333 44, fax.: +41-3823 32 01, e-mail: Hof @imt.unine.ch
}

\begin{abstract}
We compare the outdoor performance of single junction a-Si:H PV-modules which were mounted in three different ways. One was thermally well isolated against convection and radiation losses in order to reach maximum operating temperatures. A second one was fixed onto a radiator to keep its temperature as close as possible to the one of the air. A third one served as reference and was mounted with an open back side.

By the different mountings the operating temperature of the modules could be strongly influenced. Although a high operating temperature results in a reduced $V_{O C}$, it turned out to be beneficial for the effective energy conversion in the long term due to a strongly reduced Staebler-Wronski degradation.
\end{abstract}

\section{INTRODUCTION}

The temperature coefficient of the conversion efficiency of photovoltaic modules is generally negative. This is mainly due to an increase of the dark current with rising temperature which results in a reduction of the $V_{o c}$ (e.g. $-0.4 \%$ per ${ }^{\circ} \mathrm{C}$ for crystalline silicon). The actual power generation of a given module is therefore critically affected by the way this module is mounted. In order to keep the operating temperature of PV modules as low as possible one should let the air circulate around them freely. This requirement implies that special care has to be taken if the modules are to be integrated onto building

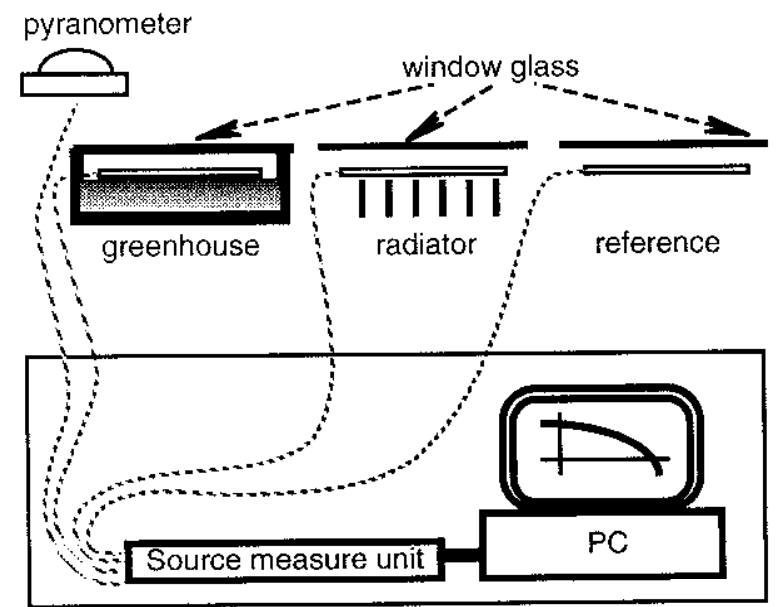

Fig. 1: Illustration of the experimental set up. In order to match intensity and spectral distribution of the illumination all the modules have been covered with the same kind of window glass plate.

\begin{tabular}{|c|c|}
\hline $\begin{array}{l}\text { module } 1: \\
\text { (greenhouse) }\end{array}$ & $\begin{array}{l}\text { It is mounted into a "greenhouse" box, of } \\
\text { which we made the transparent front side } \\
\text { out of window glass. The back side and } \\
\text { the borders are thermally well isolated } \\
\text { against radiation and convection losses } \\
\text { (operating temperatures rising up to > } \\
100^{\circ} \mathrm{C} \text { ). }\end{array}$ \\
\hline $\begin{array}{l}\text { module } 2: \\
\text { (radiator) }\end{array}$ & $\begin{array}{l}\text { Its back is brought into intimate thermal } \\
\text { contact with a radiator (operating } \\
\left.\text { temperature does not exceed } 45^{\circ} \mathrm{C}\right)\end{array}$ \\
\hline $\begin{array}{l}\text { module } 3: \\
\text { (reference) }\end{array}$ & $\begin{array}{l}\text { This module serves as reference. it is } \\
\text { mounted with the back open to the air } \\
\text { (operating temperature reaches up to } \\
60^{\circ} \mathrm{C} \text { ) }\end{array}$ \\
\hline
\end{tabular}

Table 1. Description of the three different mountings.

facades for example.

For a-Si:H solar cells it is on one hand well known that the sensitivity of the $V_{O C}$ on the operating temperature is less important when compared to crystalline silicon. On the other hand it has been observed that operation at higher temperatures leads to a higher "stabilised" fill factor since the Staebler-Wronski degradation is particularly pronounced if the modules are exposed to intense illumination at low temperatures [2]. It is however a controversial subject [3-6], whether these two competing effects result in the long term in an apparently positive or negative temperature coefficient of the efficiency.

Under outdoor conditions a further complication arises by the fact, that the temperature as well as the illumination intensity may change arbitrarily. Predictions are then even more difficult. In order to estimate the importance of these two contributions of temperature related effects on conversion efficiency we performed an experiment in which we monitored the IV-curves of differently mounted solar modules during nine months of outdoor exposure.

\section{EXPERIMENTAL}

In order to obtain the most strongly marked effects we chose single junction modules (Sanyo TF100100PL, $10^{*} 10 \mathrm{~cm}^{2}$ on glass). These modules are not encapsulated but just protected by a polymeric coating which simplifies the temperature measurement of the cells. Prior to the outdoor experiments we performed temperature coefficient measurements that allowed us to verify that the three selected modules show a comparable behaviour. 


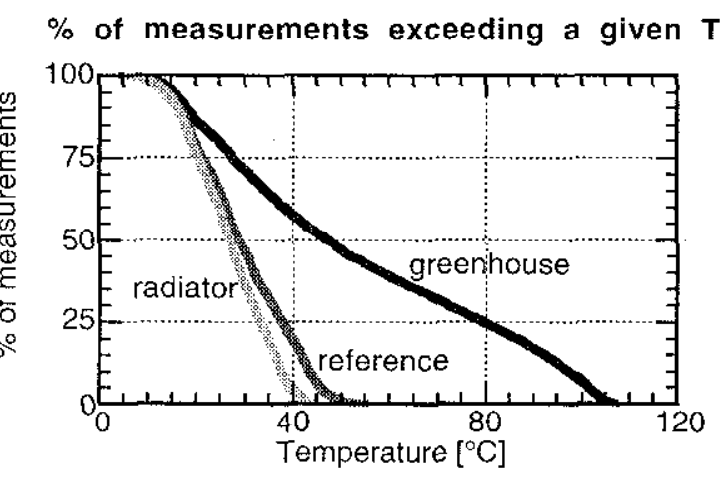

Fig. 2: Statistical distribution of the temperature values measured on the modules during daytime (for the period between June and September). One can clearly see that the greenhouse module has seen temperatures up to $105^{\circ} \mathrm{C}$. Also visible is the fact that while module 3 was heated up to $60^{\circ} \mathrm{C}$, for module 2 the temperature never exceeded $45^{\circ} \mathrm{C}$. On the average the greenhouse module was about $25^{\circ} \mathrm{C}$ hotter than the reference.

These modules were then mounted accordingly to the description in table 1 . To have the same intensity as well as the same spectral distribution of the illumination on all the cells, we fixed the same type of glass plate, as was used for the window of the green house, as a filter over the second and third module. The temperature of each module was measured by means of a thermistor (PT 100) which was glued on the protective coating on the back. The illumination was determined by a silicon solar cell pyranometer. Every $300 \mathrm{~s}$, a measurement of the illumination, of the IV-characteristics, as well as of the temperature of the corresponding module were automatically recorded for all the modules. The modules were otherwise kept under open circuit conditions.

\section{RESULTS AND DISCUSSION}

\section{Effects of mounting on module temperature}

First of all we noted that the desired effects on the actual module temperature could indeed be obtained by

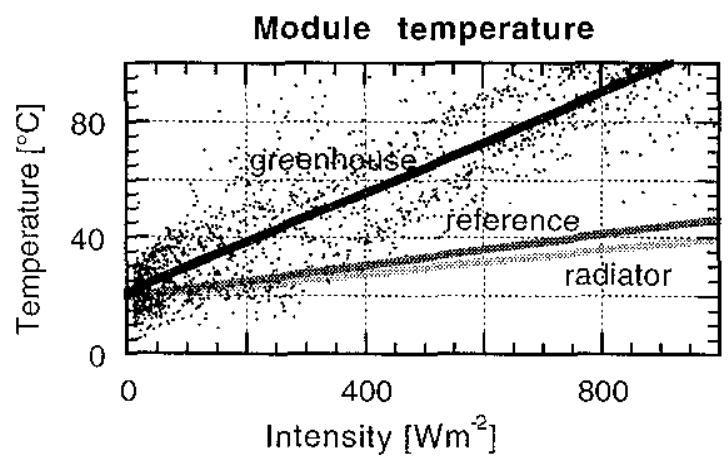

Fig. 3: Module temperature as a function of the incoming light intensity. Thermal inertia of the modules together with arbitrary changes of the intensity result in a strong scattering of the data (plotted here only for the greenhouse!). The shown lines are best linear fits.

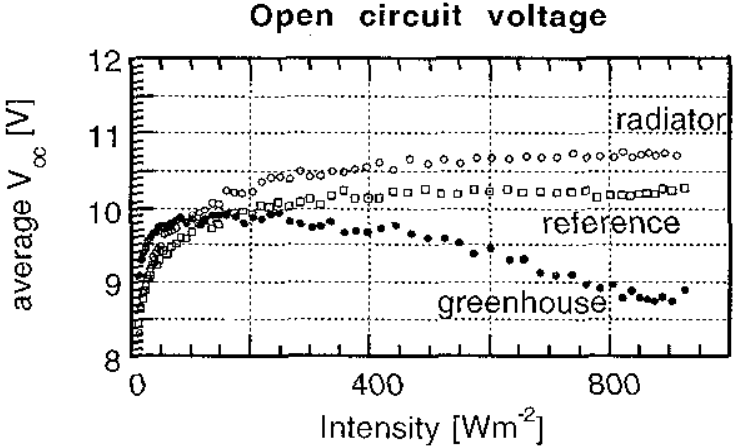

Fig. 4: Open circuit voltages of the three modules depending on the light intensity (shown are average values measured at a given intensity). One observes that the $V_{O C}$ of the greenhouse module tends to decrease for strong illumination. The inverse holds for the modules which operated at comparably low temperatures. (The mismatch between the radiator module and the reference can only partially be explained with the former's lower temperature.)

the above described passive means. We observed (see Fig. 2) that during the period of June to September $25 \%$ of all measurements done on the greenhouse module exceeded $80^{\circ} \mathrm{C}$. It reached even temperatures of up to $105^{\circ} \mathrm{C}$, while the temperature range of the reference module was limited to $60^{\circ} \mathrm{C}$ and was even restricted to $45^{\circ} \mathrm{C}$ for the module fixed on the radiator.

The heating of the modules is of course directly connected with the incoming light intensity. This is visualised by a plot of the module temperature as a function of the light intensity (see Fig. 3). Since the modules (especially the greenhouse module) do have however a thermal inertia and since the illumination intensity may be subject to rapid changes due to the shading by the clouds passing by (especially in Switzerland) one gets strong scattering of the data. In Fig. 3 we show therefore only the best linear fits to the measured data. This way the very strong dependence of the module temperature on the illumination in the case of the greenhouse could be represented as well as the reduced dependence for the radiator module.

\section{Effects of mounting on $\mathrm{v}_{\mathrm{oc}}$}

The immediately observable effect of these temperature differences is a different behaviour of the $V_{O C}$ versus light intensity characteristics (see Fig. 4). For constant temperatures one would expect it to increase (in a first order approximation) logarithmically with the illumination. Since the module temperature however depends also directly on the incoming radiation intensity one gets a superposition of two contrary effects. The second effect acts with a longer time constant and is responsible for the scattering of the data taken during unstable weather conditions. In the case of the relatively cool modules the $V_{o c}$ remains more or less constant at high intensities. For the greenhouse module one observes an actual decrease of the $V_{o c}$ at higher intensities. This is due to remarkably higher temperatures at these high intensities (see Fig. 3). 


\section{Evolution of $\mathrm{I}_{\mathrm{sc}}$}

While considering the short circuit current (normalised by the incident intensity) one again observes a relatively strong scattering of the data. This time the variations may be mainly attributed to the fact that the light intensity could not be measured simultaneously but only immediately after the acquisition of the IV-curve (which generally took several seconds). If plotted against the time one can nevertheless observe the tendency of a slight degradation of the $\mathrm{I}_{\mathrm{sc}}$ for all the modules (Fig. 5). The higher operating temperature of the greenhouse module does not influence the stability of its current.

\section{Evolution of FF}

A strong effect of the operating temperature on stability is however observed in the evolution of the fill factor. For the reference as well as for the radiator module we observed a strong FF degradation during the nine months of exposure. This effect is strongly reduced in the case of the greenhouse module (Fig. 6). Its fill factor remains during the whole period above 0.68 , which is about $20 \%$ higher than the value of the reference module.

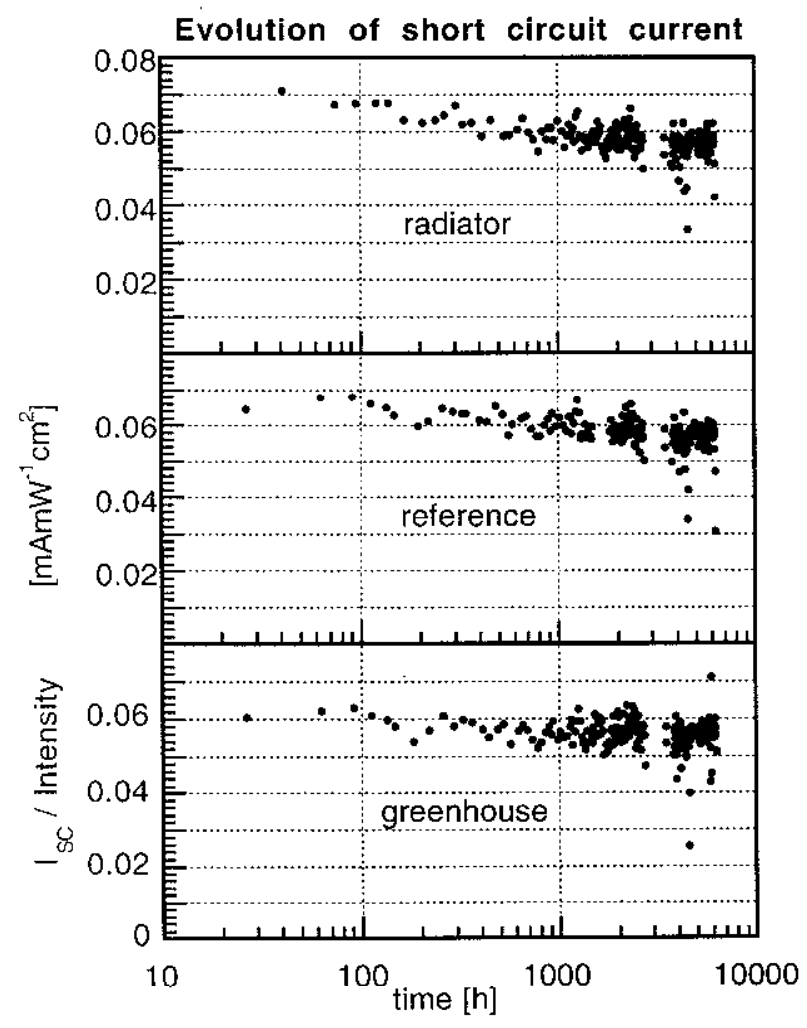

Fig. 5: Temporal evolution of the short circuit currents (normalised by the incident intensity $\left[\mathrm{mAmW}^{-1} \mathrm{~cm}^{2}\right]$ ) of the three modules. In all the three cases one observes a slight dearadation as a function of time.

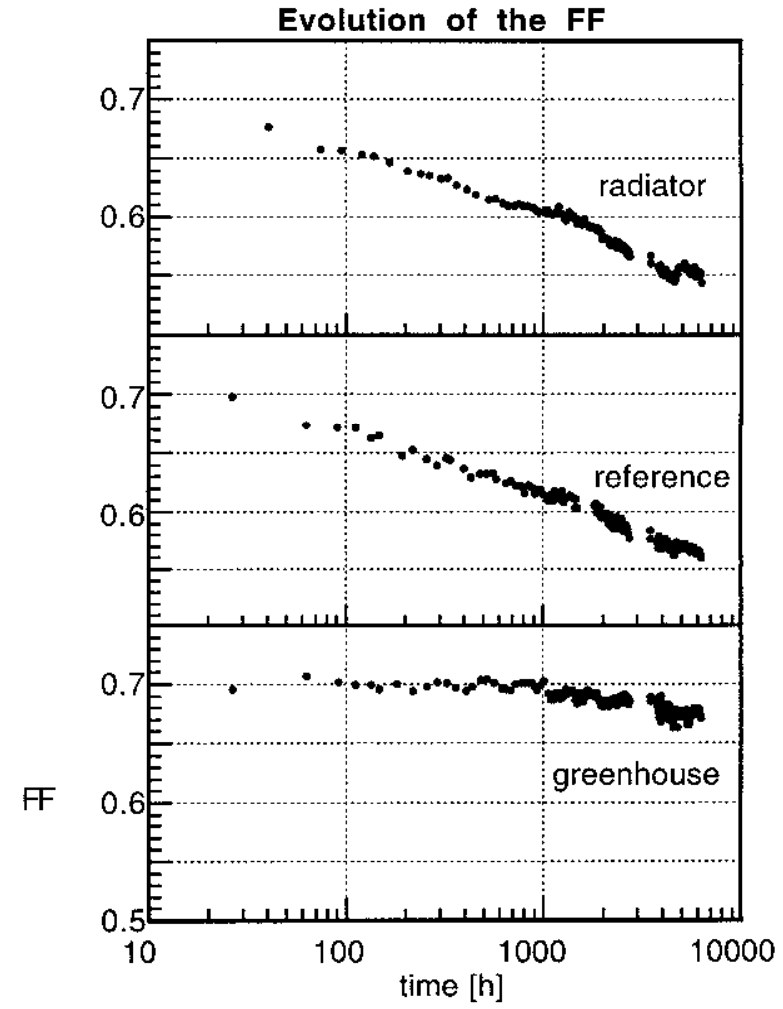

Fig. 6: Temporal evolution of the fill factor. Clearly the greenhouse module shows an almost stable fill factor, whereas the other two modules strongly degrade.

\section{Effect of mounting on the efficiency}

In order to discriminate between the importance of the temperature related effects on the $V_{o c}$ and on the FF we consider in Fig. 7 the average efficiency of the three modules as a function of time. One clearly observes that

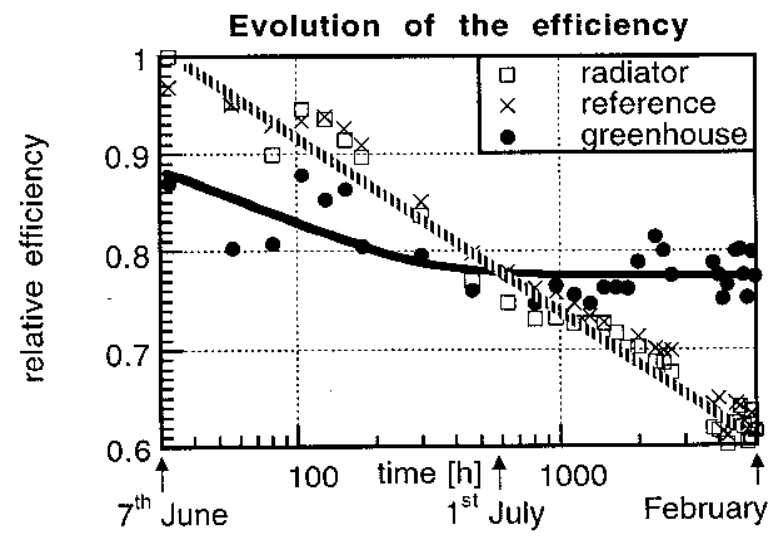

Fig. 7: Evolution of the average efficiency (normalised by the highest initial efficiency which was approximately $5 \%$ ). The time scale refers to the beginning of the experiment. The greenhouse module had the lowest efficiency during the first days. Later on it was clearly the leader of the three. 

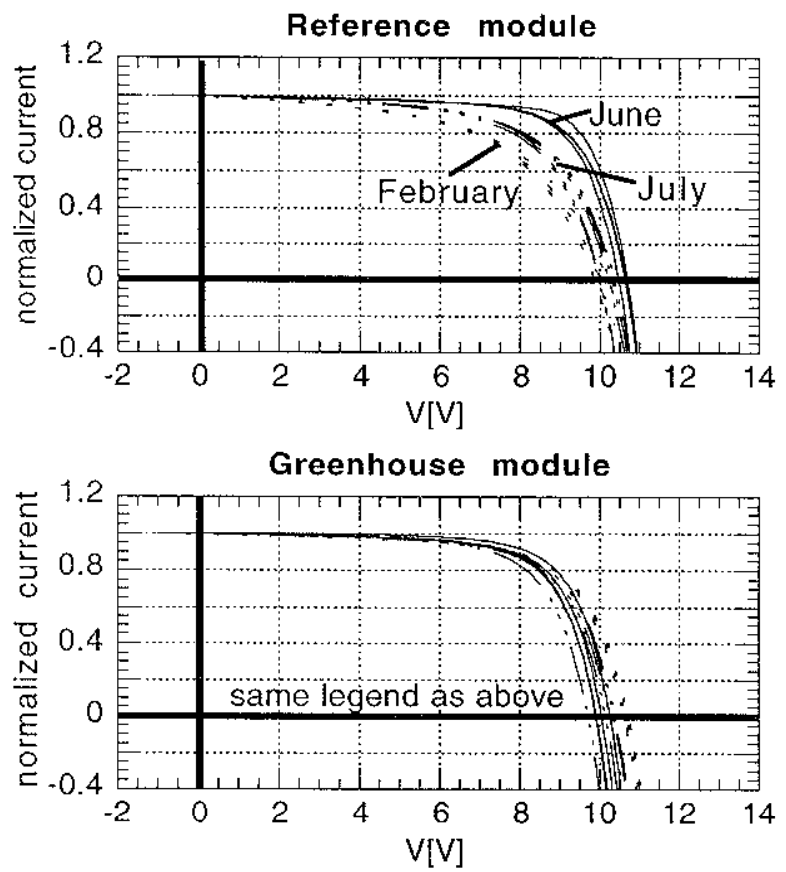

Fig. 8: Normalised IV-curves of the greenhouse module compared to the reference measured at intensities of approximately $200 \mathrm{Wm}^{-2}$. The $\mathrm{FF}$ after degradation is clearly higher for the greenhouse module in comparison with the reference.

the combination of these different effects leads in the short term to lower efficiencies for the greenhouse module due to a relatively low $V_{O C}$ at high intensities. After some time however, the better stability of this module (with respect to the Staebler-Wronski effect) overcompensates these $V_{O C}$ losses. The greenhouse module shows then the highest conversion efficiency (see also the IV-curves shown in Fig. 8).

Outdoor experiments with a-Si:H solar cells show generally that their efficiency undergoes seasonal variations [6]. Thereby one observes a minimum of the efficiency during wintertime. Our experiment now indicates, that the performance of a module which is mounted in a way to reach high temperatures is better during wintertime when compared to others. This is certainly an advantage of this type of mounting.

We also observed in our case that the efficiency of the greenhouse module was inferior only during about one month after exposure. Its average performance over the period of the experiment was therefore also the highest.

The gain due to reduced degradation during wintertime depends of course on the type of module which is used. Since the efficiency losses due to $V_{o c}$ reduction during summer amount in our configuration to about $15 \%$, we expect no gain anymore, if a given module degrades under normal conditions less than approximately this same amount.

The improvement of the stability will also depend on the increase of temperature which can be achieved by a given type of mounting. Our experiment with the greenhouse presents certainly an extreme case which could be realised in a hybrid solar module as described for example in [8]. A configuration making use of the above described effects which is more likely to be implemented is the integration of amorphous solar modules directly on a facade where no air cooling of the back side is provided.

\section{CONCLUSION}

We have compared the module performances of a-Si:H single junction modules in three different situations characterised by identical illumination conditions but different temperature ranges. In the long term a mounting by which high operating temperatures of the modules are obtained has a beneficial effect on the conversion efficiency. This makes amorphous silicon solar modules an interesting candidate for facade integration (simplified implementation since air cooling is not required) or as an absorber for hybrid (electrical and thermal) energy conversion systems.

\section{ACKNOWLEDGEMENT}

This work was supported by the Swiss Federal Office of Education and Science under the grant (OFES 993.0184).

\section{REFERENCES}

[1] M. A. Green, "Solar Cells", University of New South Wales, 1992 , p. 92

[2] B. von Roedern, "New insights into the StaeblerWronski degradation mechanism from analyses of solar cell and module degradation data", Thirteenth European PVSEC, 1995, pp.1672-1676

[3] Y. Ichikawa et al., "Large-area amorphous silicon solar cells with high stabilised efficiency and their fabrication technology", Twenty third IEEE PVSC, 1993, pp. 27-33

[4] E. D.Dunlop, "Modelling amorphous photovoltaic arrays using positive temperature coefficients and standard ambient parameters", Thirteenth European PVSEC, 1995, pp. 623-626

[5] P. D. Lund et al., "Advanced a-Si PV systems approaches to building integration", Thirteenth European PVSEC, 1995, 2227-2230

[6] C. M. Whitaker et al., "Effects of irradiance and other factors on PV temperature coefficients", Twenty first IEEE PVSC, 1991, pp. 608-613

[7] M. Camani et al., "TISO: Tests of reliability on crystalline and amorphous silicon modules", Thirteenth European PVSEC, 1995, pp. 2345-2348

[8] A. Ricaud et P. Roubeau, "'Capthel', a 66\% efficient hybrid solar module and the 'Ecothel' cogeneration solar system", Twenty fourth IEEE PVSC, 1994, pp. 1012-1015 